\title{
Accuracy of GPS Devices for Measuring High-intensity Running in Field-based Team Sports
}

Authors

Affiliations

\author{
E. Rampinini ${ }^{1}$, G. Alberti ${ }^{2}$, M. Fiorenza ${ }^{3}$, M. Riggio ${ }^{4}$, R. Sassi ${ }^{5}$, T. O. Borges ${ }^{6}$, A. J. Coutts
}

Affiliation addresses are listed at the end of the article

\author{
Key words \\ - soccer \\ - team sport \\ metabolic power \\ - acceleration \\ training load monitoring
}

accepted after revision

May 27, 2014

Bibliography DOI http://dx.doi.org/ 10.1055/s-0034-1385866 Published online: 2014 Int J Sports Med (c) Georg Thieme Verlag KG Stuttgart · New York ISSN 0172-4622

Correspondence

Prof. Ermanno Rampinini

S. S. MAPEI srl

Human Performance Laboratory

via Busto Fagnano 38

Olgiate Olona

Italy 21057

Tel.: + 39/0331/575 757

Fax: $+39 / 0331 / 575728$

physiolab@mapeisport.it

\section{Abstract \\ $\nabla$}

We compared the accuracy of 2 GPS systems with different sampling rates for the determination of distances covered at high-speed and metabolic power derived from a combination of running speed and acceleration. 8 participants performed 56 bouts of shuttle intermittent running wearing 2 portable GPS devices (SPI-Pro, GPS $-5 \mathrm{~Hz}$ and MinimaxX, GPS $-10 \mathrm{~Hz}$ ). The GPS systems were compared with a radar system as a criterion measure. The variables investigated were: total distance (TD), high-speed distance $\left(\mathrm{HSR}>4.17 \mathrm{~m} \cdot \mathrm{s}^{-1}\right)$, very high-speed distance $\left(\right.$ VHSR $\left.>5.56 \mathrm{~m} \cdot \mathrm{s}^{-1}\right)$, mean power (Pmean), high metabolic power $\left(\mathrm{HMP}>20 \mathrm{~W} \cdot \mathrm{kg}^{-1}\right)$ and very

\section{Introduction}

$\nabla$

Many team-sports (e.g. soccer, rugby, Australian football) require the ability to sustain high-intensity, intermittent exercise [18]. The most common method to quantify high-intensity activities during training or matches is to determine the distance covered or the time spent above a fixed running speed (e.g. distance covered or time spent with running speed above $4.17 \mathrm{~m} \cdot \mathrm{s}^{-1}$, high-velocity activity) $[2,19,22]$. However, the ability to rapidly accelerate and decelerate (even without reaching a high level of running speed) may be considered important for team-sports performance [17]. Recently, a new method for the quantification of the high-intensity activities has been proposed, which also takes into account the phases of accelerated and decelerated running $[10,20]$. This new approach is based on a theoretical model [8] that allows the energetic cost of accelerations and decelerations during running to be calculated, and consequently allows the derivation of metabolic power output during intermittent running activities such as high metabolic power $\left(\mathrm{VHMP}>25 \mathrm{~W} \cdot \mathrm{kg}^{-1}\right)$. GPS $-5 \mathrm{~Hz}$ had low error for TD (2.8\%) and Pmean (4.5\%), while the errors for the other variables ranged from moderate to high (7.5-23.2\%). GPS$10 \mathrm{~Hz}$ demonstrated a low error for TD (1.9\%), HSR (4.7\%), Pmean (2.4\%) and HMP (4.5\%), whereas the errors for VHSR (10.5\%) and VHMP (6.2\%) were moderate. In general, GPS accuracy increased with a higher sampling rate, but decreased with increasing speed of movement. Both systems could be used for calculating TD and Pmean, but they cannot be used interchangeably. Only GPS- $10 \mathrm{~Hz}$ demonstrated a sufficient level of accuracy for quantifying distance covered at higher speeds or time spent at very high power.

team sports. The application of this method has been suggested to be superior to traditional time-motion analysis variables as it provides a better estimate of the overall energy demands of team sport activities.

Global positioning system (GPS) technology has rapidly advanced in recent years and has become a common method for assessing the physical demands of training and competition in fieldbased team sports [1]. Several studies have investigated the validity and reliability of GPS devices for measuring movements and speeds $[7,11,16]$, but direct comparison between these studies is difficult because of the different methods of investigation [1]. Nevertheless, it has been shown that the sample rate of the devices, speed and effort duration and nature of the exercise task affect the accuracy and the reliability of GPS. Specifically, it appears that validity improves with higher sampling rate, while reliability decreases in tasks that require regular changes of direction and brief accelerations $[7,11,16]$. Indeed, a recent investigation demonstrated that the latest GPS units which sample at $10 \mathrm{~Hz}$ were 
sufficiently accurate to quantify the acceleration and deceleration running phases in team sports [25]. However, the theoretical model for the metabolic power determination was developed based on running speed data collected using a radar system [8]. Using GPS data to estimate metabolic power has significant advantages for team sports compared to the use of radar, as the radar measures only provide sufficient accuracy during straight line running. A recent study used GPS data sampled at $15-\mathrm{Hz}$ and subsequently averaged out to $5-\mathrm{Hz}$ to assess the training demands in top professional soccer players using the metabolic power model [10]. To date, however, no study has attempted to verify the accuracy of the GPS systems for this purpose.

The aim of this study was therefore to compare the accuracy of 2 GPS systems with different sampling rates for the quantification of the distance covered at high-speed as well as for the determination of metabolic power.

\section{Material \& Methods}

$\nabla$

8 sub-elite young male football players (age: $15 \pm 1$ years, body mass: $59.3 \pm 9.1 \mathrm{~kg}$ and height: $173 \pm 7 \mathrm{~cm}$ ) were involved in the study. The parents of the subjects provided written informed consent prior to participation in the study, which was approved by the Independent Institutional Review Board of Mapei Sport Research Centre in accordance with the Helsinki Declaration and meets the ethical standards of the journal [13].

To determine the accuracy of 5 and $10 \mathrm{~Hz}$ GPS, each subject completed 7 bouts of an intermittent running exercise which simulated very intense phases of a soccer match (i.e., characterized by changes in activity every $\sim 5 \mathrm{~s}$ and regular speed entries $\left.>4 \mathrm{~m} \cdot \mathrm{s}^{-1}\right)[2,19]$. The 7 bouts consisted of $70 \mathrm{~m}(35+35 \mathrm{~m})$ of self-paced, straight line intermittent shuttle runs over a marked course involving walking, jogging, accelerations and decelerations during running at different intensities ( $\bullet$ Fig. 1, panel a). Of the 7 bouts completed by the participants, 4 were comprised of 3 bouts of the $70 \mathrm{~m}$ course (for a total of $210 \mathrm{~m}$ ) in addition to 3 bouts of the course 4 times $(280 \mathrm{~m})$. A straight line running course was used to ensure accuracy of the criterion radar measure. In total, 56 bouts were undertaken but, due to technical problems (e.g. loss of radar data or the GPS systems switching off during the trials), only 47 trials were considered for the analysis.

Instantaneous running speed was recorded using a radar system (Stalker ATS, Radar Sales, Minneapolis, MN, US) sampling at $32 \mathrm{~Hz}$, which was considered the criterion measure because this system has a high level of accuracy in the running speed measure [4] and the metabolic power model was originally developed using data collected with this apparatus. Raw speed data were filtered using a zero-lag Butterworth filter. The radar device was positioned $2 \mathrm{~m}$ behind the starting point at a height of $1.2 \mathrm{~m}$. In addition, participants wore 2 reflective panels (one on the back and one on the abdomen) to provide an appropriate reflective surface for the radar system. The accuracy and reliability of the system was previously reported and can be considered as very high $[4,5]$.

During the entire test session players wore 2 portable GPS devices (SPI-Pro GPSports System, $5 \mathrm{~Hz}$, Canberra, Australia, GPS-5 Hz and MinimaxX v4.0 Catapult Innovations, $10 \mathrm{~Hz}$, Melbourne, Australia, GPS-10 Hz) positioned on the upper back in a custom-made vest. The antennae of each unit were exposed to allow clear satellite reception. The mean number of satellites connected during data collection was $12.3 \pm 0.3$ (units range:

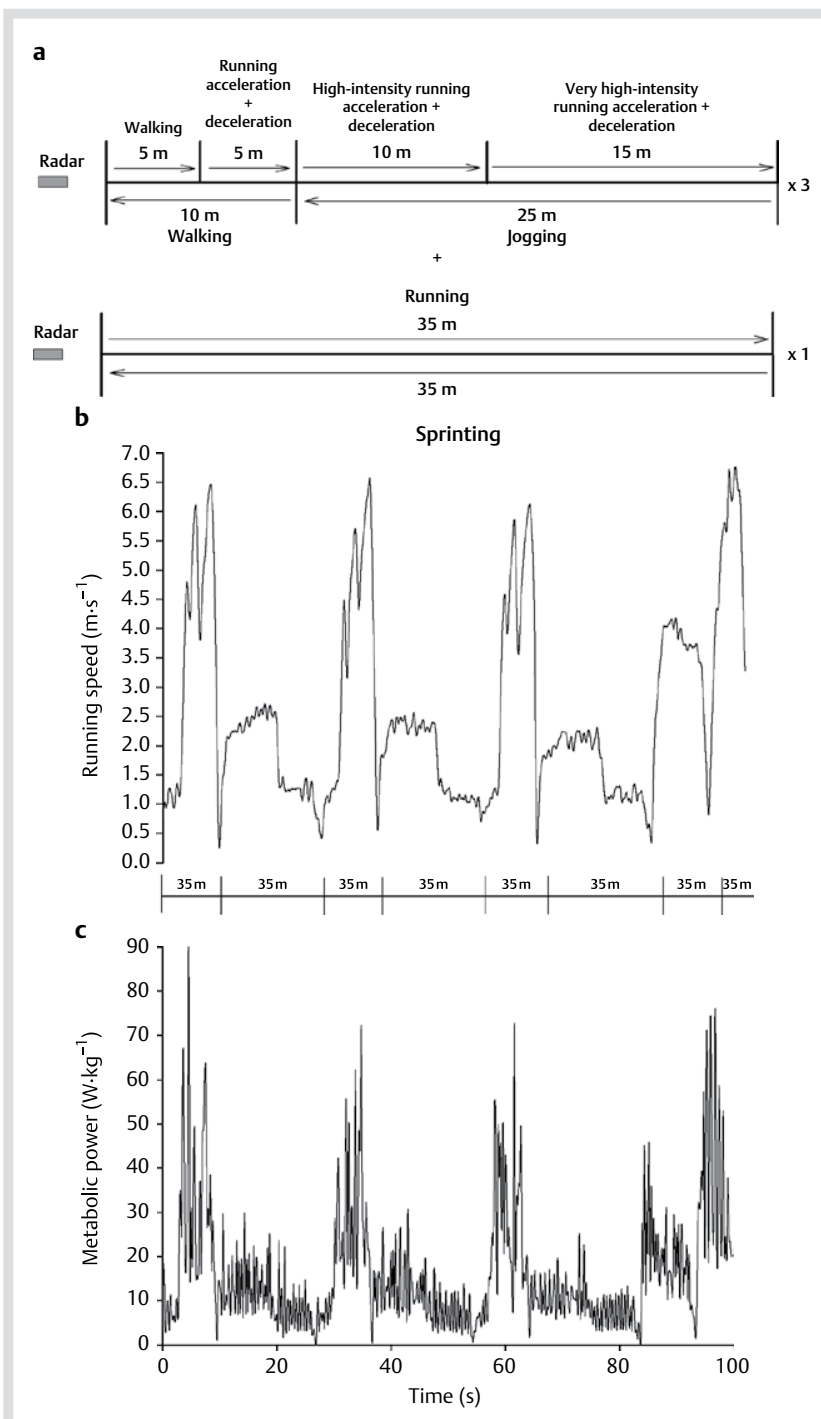

Fig. 1 Schematic representing the activities performed during each bout of the intermittent shuttle running (panel a) and an example of running speed measurement (panel b) and metabolic power calculation (panel c) using the radar system.

12.0-12.9), while the mean horizontal dilution of position was $0.9 \pm 0.1$ (units range: $0.8-1.1$ ).

For each bout, data recorded using each system were exported and placed in a customised Microsoft Excel spreadsheet (Microsoft, Redmond, USA) for the calculation of the selected variables: total distance covered (TD); high-speed running distance (running speed $>4.17 \mathrm{~m} \cdot \mathrm{s}^{-1}$, HSR); very high-speed running distance (running speed $>5.56 \mathrm{~m} \cdot \mathrm{s}^{-1}$, VHSR). Furthermore, energy cost (EC) and instantaneous metabolic power $\left(\mathrm{P}_{\text {met }}\right)$ were estimated using the equation proposed by Di Prampero et al. [8] and then modified by Osgnach et al. [20]:

\section{$\mathrm{EC}=\left(155.4 \cdot \mathrm{ES}^{5}-30.4 \cdot \mathrm{ES}^{4}-43.3 \cdot \mathrm{ES}^{3}+46.3 \cdot \mathrm{ES}^{2}+19.5 \cdot \mathrm{ES}+3.6\right) \cdot$ $\mathrm{EM} \cdot \mathrm{KT}$}

where EC is the energy cost of accelerated running on grass $\left(\mathrm{J} \cdot \mathrm{kg}^{-1} \cdot \mathrm{m}^{-1}\right)$; ES is the equivalent slope $\left(\mathrm{ES}=\tan \left(90\right.\right.$-arcan $\left.\mathrm{g} / \mathrm{a}_{\mathrm{f}}\right)$, $\mathrm{g}=$ Earth's acceleration of gravity, $\mathrm{a}_{\mathrm{f}}=$ forward acceleration); EM is the equivalent body mass $\left(\mathrm{EM}=\left(\mathrm{a}_{\mathrm{f}}^{2} / \mathrm{g}^{2}+1\right)^{0.5}\right)$; and $\mathrm{KT}$ is a con$\operatorname{stant}(\mathrm{KT}=1.29)$. 
Consequently, $\mathrm{P}_{\text {met }}\left(\mathrm{W} \cdot \mathrm{kg}^{-1}\right)$ was calculated multiplying EC by running speed $\left(\mathrm{V}, \mathrm{m} \cdot \mathrm{s}^{-1}\right)$ :

$\mathrm{P}_{\text {met }}=\mathrm{EC} \cdot \mathrm{V}$

The metabolic power parameters considered were: mean metabolic power (Pmean); time spent at high metabolic power (metabolic power $>20 \mathrm{~W} \cdot \mathrm{kg}^{-1}, \mathrm{HMP}$ ) and time spent at very high metabolic power (metabolic power $>25 \mathrm{~W} \cdot \mathrm{kg}^{-1}, \mathrm{VHMP}$ ). For each bout, the first and last $5 \%$ of the data were excluded from analysis to prevent the edge effect due to the filtering algorithm. $\odot$ Fig. 1 depicts an example of measured running speed (panel b) and calculated metabolic power (panel c) during one bout of intermittent running using the radar system. For each bout of running, the raw radar and GPS data were aligned starting from the origin of the running speed curve.

The accuracy of the 2 GPS units for measuring the aforementioned variables was assessed comparing segmented data based on actual velocity derived from the criterion measurement tool (radar) with GPS data. Data are presented as mean \pm SD, unless stated otherwise. When a data set violated the assumption of normality, they were log transformed to reduce non-uniformity of error. A linear mixed-effects model using the "multilevel" package in $\mathrm{R}$ software was used to determine the individual responses of each dependent variable collected from different devices. The participants were included as a random effect in the model to correct for pseudoreplication. The $t$ and chi-square statistics from the linear mixed modelling were then converted into r-values and considered as the effect size (ES) [6]. The r-values were then interpreted as ES using thresholds of 0.0, 0.1, 0.3, 0.5, 0.7, 0.9 and 1 as trivial, small, moderate, large, very large, nearly perfect and perfect, respectively [15]. All of these statistical procedures were performed using the R software. Further-

Table 1 Performance variables (mean \pm SD) measured using the criterion system (radar) and the 2 GPS devices (GPS-5Hz and GPS-10 Hz) during the intermittent exercise.

\begin{tabular}{lccc} 
& Radar & GPS-5 Hz & GPS-10 Hz \\
TD $(m)$ & $228 \pm 32$ & $233 \pm 34$ & $230 \pm 35$ \\
HSR $(\mathrm{m})$ & $111 \pm 14$ & $107 \pm 14$ & $110 \pm 13$ \\
VHSR $(\mathrm{m})$ & $51 \pm 13$ & $44 \pm 17$ & $48 \pm 15$ \\
Pmean $\left(\mathrm{W} \cdot \mathrm{kg}^{-1}\right)$ & $17.8 \pm 3.4$ & $18.1 \pm 1.4$ & $16.2 \pm 1.4$ \\
\hline HMP $(\mathrm{s})$ & $22.5 \pm 3.4$ & $25.1 \pm 3.2$ & $21.9 \pm 3.2$ \\
VHMP $(\mathrm{s})$ & $16.1 \pm 2.3$ & $16.6 \pm 2.4$ & $15.0 \pm 2.2$
\end{tabular}

TD, total distance covered; HSR, distance covered at high-speed running $>4.17 \mathrm{~m} \cdot \mathrm{s}^{-1}$; VHSR, distance covered at very high-speed running $>5.56 \mathrm{~m} \cdot \mathrm{s}^{-1}$; Pmean, mean metabolic power; HMP, time spent at high metabolic power $>20 \mathrm{~W} \cdot \mathrm{kg}^{-1}$ and VHMP, time spent at very high metabolic power $>25 \mathrm{~W} \cdot \mathrm{kg}^{-1}$ more, the typical error (TE) expressed as a coefficient of variation (CV) and relative $90 \%$ confidence limits were calculated using Hopkins' spreadsheet (http://www.sportsci.org/resource/stats/ relycalc.html\#excel) [14]. The TE was considered low $<5 \%$, moderate $5-10 \%$ and high $>10 \%$. In addition, bias and relative $90 \%$ confidence limits were also calculated, and significant differences were verified through a series of paired t-test using STATISTICA (version 8.0, Tulsa, USA). Significance was set at $\mathrm{P}<0.05$.

\section{Results \\ $\nabla$}

- Table 1 reports the mean values of all selected variables calculated from the running speed data collected using the 3 systems. The mixed model analysis did not show significant differences between devices for $\mathrm{TD}-[\mathrm{t}(131)=0.6, \mathrm{p}=0.50, \mathrm{ES}=0.05$ trivial $]$, although the random variation of subjects was significant $\left[\mathrm{X}^{2}(1)=144.0, \mathrm{p}<0.0001, \mathrm{ES}=0.9\right.$ nearly perfect, $\mathrm{SD}_{\text {intercept }}=0.03$ (90\% CI 0.02-0.06), $\mathrm{SD}_{\text {slope }}=0.008(90 \%$ CI 0.004-0.01)]. There were also significant differences between devices for Pmean $[\mathrm{b}=-0.04(90 \% \mathrm{CI}-0.05$ to -0.03$), \mathrm{t}(131)=-6.0, \mathrm{p}<0.001, \mathrm{ES}=0.5$ large] with significant random variation for the subjects $\left[\mathrm{X}^{2}(2)=32.5, \mathrm{p}<0.0001, \mathrm{ES}=0.9\right.$ nearly perfect, $\mathrm{SD}_{\text {intercept }}=0.07$ (90\% CI 0.04-0.11), $\left.\mathrm{SD}_{\text {slope }}=0.02(90 \% \mathrm{CI} 0.01-0.03)\right]$. In contrast, there were no significant differences between devices for HSR $[\mathrm{t}(130)=-0.91, \mathrm{p}=0.3, \mathrm{ES}=0.07$ trivial and random variation for the subjects $\mathrm{X}^{2}(2)=1.5, \mathrm{p}=0.5$, ES $=0.4$ moderate] or HMP $[t(131)=-1.05, p=0.3, E S=0.09$ small $]$ and the random variation for both intercept and slopes $-\left[\mathrm{X}^{2}(2)=0.5, \mathrm{p}=0.8, \mathrm{ES}=0.2\right.$ small $]$. There were no significant differences between devices for VHSR - $[\mathrm{t}(131)=-1.25, \mathrm{p}=0.2, \mathrm{ES}=0.1$ trivial $]$ and random variation for the subjects $\left[\mathrm{X}^{2}(1)=0.4, \mathrm{p}=0.5, \mathrm{ES}=0.2\right.$ small], while there were significant differences between devices for VHMP $[b=-0.03 \quad(90 \% C I-0.05$ to-0.01), $\mathrm{t}(131)=-3.1, \quad \mathrm{p}=0.0027$, $\mathrm{ES}=0.2$ small], with significant random variation for the subjects $\left[\mathrm{X}^{2}(1)=9.2, \mathrm{p}=0.0024, \mathrm{ES}=0.7\right.$ very large, $\mathrm{SD}_{\text {intercept }}=0.01(90 \% \mathrm{CI}$ $0.0006-0.31), \mathrm{SD}_{\text {slope }}=0.01(90 \%$ CI $\left.0.004-0.02)\right]$.

Typical errors and systematic biases between GPS systems and criterion measure are presented in 0 Table 2. The GPS $-5 \mathrm{~Hz}$ showed a low TE as CV for TD (2.8\%) and Pmean (4.5\%). The same system demonstrated a moderate TE as CV for HSR (7.5\%) and HMP (9.0\%), while TE as CV was high for VHSR (23.2\%) and VHMP (11.6\%). The GPS $-10 \mathrm{~Hz}$ showed low TE as CV for TD (1.9\%), HSR (4.7\%), Pmean (2.4\%) and HMP (4.5\%). For the same system the TE as CV was high for VHSR (10.5\%) and moderate for VHMP (6.2\%). In addition, the GPS-5Hz significantly overestimated TD (1.8\%) and HMP (11.7\%), while significantly underestimating HSR $(-4.0 \%)$ and VHSR $(-17.8 \%)$. The GPS $-10 \mathrm{~Hz}$

\begin{tabular}{|lcccc} 
& \multicolumn{2}{c}{ TE as CV (\%) } & \multicolumn{2}{c}{ Bias (\%) } \\
& Radar vs. GPS-5 Hz & Radar vs. GPS-10 Hz & Radar vs. GPS-5 Hz & Radar vs. GPS-10 Hz \\
\hline TD & $2.8(2.3 ; 3.3)$ & $1.9(1.6 ; 2.3)$ & $1.8(0.8 ; 2.7)^{*}$ & $0.6(-0.1 ; 1.3)$ \\
\hline HSR & $7.5(6.4 ; 9.1)$ & $4.7(4.0 ; 5.8)$ & $-4.0(-6.4 ;-1.6)^{*}$ & $-1.1(-2.7 ; 0.5)$ \\
VHSR & $23.2(19.5 ; 28.7)$ & $10.5(9.0 ; 12.5)$ & $-17.8(-23.5 ;-11.6)^{* *}$ & $-7.3(-10.4 ;-4.0)^{*}$ \\
Pmean & $4.5(3.8 ; 5.5)$ & $2.4(2.1 ; 2.9)$ & $1.5(0.1 ; 3.1)$ & $-8.7(-9.5 ;-8.0)^{* *}$ \\
HMP & $9.0(7.6 ; 10.9)$ & $4.5(3.8 ; 5.4)$ & $11.7(8.4 ; 15.0)^{*}$ & $-2.7(-4.2 ;-1.2)^{*}$ \\
\hline VHMP & $11.6(9.8 ; 14.1)$ & $6.2(5.3 ; 7.6)$ & $3.3(-0.5 ; 7.3)$ & $-7.1(-9.0 ;-5.1)^{*}$
\end{tabular}

TE, typical error; TD $(m)$, total distance covered; HSR $(m)$, distance covered at high-speed running speed $>4.17 \mathrm{~m} \cdot \mathrm{s}^{-1} ; \mathrm{VHSR}(\mathrm{m})$, distance covered at very high-speed running speed $>5.56 \mathrm{~m} \cdot \mathrm{s}^{-1} ;$ Pmean $\left(\mathrm{W} \cdot \mathrm{kg}{ }^{-1}\right)$, mean metabolic power; HMP (s), time spent at high metabolic power $>20 \mathrm{~W} \cdot \mathrm{kg}^{-1}$ and VHMP (s), time spent at very high metabolic power $>25 \mathrm{~W} \cdot \mathrm{kg}^{-1}$. Significant bias; ${ }^{*}, \mathrm{p}<0.01$; ${ }^{* *}, \mathrm{p}<0.001$
Table 2 Typical error as a CV ( $90 \%$ confidence limits) and percent bias $(90 \%$ confidence limits) for performance variables comparing the 2 GPS systems with the criterion system (radar). 
significantly underestimated VHSR $(-7.3 \%)$, Pmean $(-8.7 \%)$, $\operatorname{HMP}(-2.7 \%)$ and VHMP ( $-7.1 \%)$.

\section{Discussion \\ $\nabla$}

The purpose of the present study was to examine the accuracy of 2 GPS devices ( 5 and $10 \mathrm{~Hz}$ sample rate) for the quantification of the high-intensity activities in field-based team sports. The main finding was that GPS $-10 \mathrm{~Hz}$ was generally more accurate than GPS-5Hz, while both systems showed greater error for the highest running speed and power categories (i.e., VHSR and VHMP).

Consistent with previous research $[7,9,16]$, higher sampling rates decreased the error from the criterion distance. In fact, GPS- $10 \mathrm{~Hz}$ showed between $30-50 \%$ lower error in the determination of TD, HSR and VHSR compared with GPS-5Hz. Despite this, the TE as a CV of GPS- $10 \mathrm{~Hz}$ for VHSR remained quite high, suggesting that caution be taken when interpreting the data, regardless of sampling rate. On the contrary, the accuracy in the measure of the distance covered at VHSR is not adequate for detecting small changes with the GPS-5Hz. In addition, caution should also be applied when interpreting HSR data collected with a $5 \mathrm{~Hz}$ GPS system. When taken in conjunction with previous observations $[3,21]$ the present results suggest that GPS systems may not be accurate enough to measure some very high-speed running such as brief single efforts $>5.56 \mathrm{~m} \cdot \mathrm{s}^{-1}$.

In addition to high-speed running [19], the ability to rapidly accelerate and decelerate may be considered important for team-sport performance [17]. Recently, a new theoretical model for the quantification of accelerated and decelerated running has been proposed that allows the metabolic power produced by the athletes to be estimated [8]. While this model has several limitations (i.e., the assumption that the overall mass of the athlete is located at the centre of mass of the body and the energy expenditure associated with the internal work is similar during uphill running and sprinting; and, not accounting for the energy cost of specific activities like jumping, kicking, tackling and dribbling the ball), the present study is the first to have established the accuracy of GPS systems for this purpose. Although the mixed model analysis demonstrated significant differences between devices for Pmean and VHMP, the TE as CV related to the mean metabolic power produced during each bout of high-intensity intermittent exercise ( $4.5 \%$ for GPS-5Hz and $2.4 \%$ for GPS- $10 \mathrm{~Hz}$ ) was acceptable for both GPS systems. This suggests that it is possible to apply the new energetic model for the quantification of accelerated and decelerated running using the raw GPS speed data, but it is not possible to use the different systems interchangeably. Furthermore, only the GPS $-10 \mathrm{~Hz}$ provided an acceptable error for the determination of $\operatorname{HMP}(4.5 \%)$ and VHMP (6.2\%). In fact, the GPS-5Hz demonstrated an especially low level of accuracy for the same variables (HMP, 9.0\% and VHMP, 11.6\%). The present results partly confirm previous findings by Varley et al. [25], who suggested that $10 \mathrm{~Hz}$ GPS yielded a higher level of accuracy for instantaneous velocity compared to $5 \mathrm{~Hz}$ GPS. However, considering the increase in the TE as a CV from HMP to VHMP ( 30-40\%) reported for both the 5 and 10-Hz GPS systems, the results of the present study calls into question the efficacy of using either system to quantify distances covered at power higher than $\sim 30 \mathrm{~W} \cdot \mathrm{kg}^{-1}$.
Consistent with several previous studies $[7,9,16]$, we observed significant under or overestimation in several variables (TD, HSR, VHSR, HMP for GPS-5Hz and VHSR, Pmean, HMP and VHMP for GPS-10Hz) with both systems. Taken collectively, these findings show that data collected with different GPS systems should not be compared directly. Moreover, we also recommend that GPS data should not be used interchangeably with other motionanalysis systems (e.g. ProZone, Amisco etc.) as differences in absolute distances covered in match play has previously been reported $[12,23]$. Based on the results of the mixed model analysis, this is particularly relevant when the metabolic power produced by the athletes is estimated applying the theoretical model proposed by Di Prampero et al. [8]. Furthermore, in the present study the accuracy of each GPS system was determined using a single unit of each model. However, in practice, the activity demands of several players from the same team are usually collected, which dictates that data from different GPS units are compared (i.e., inter-unit variability). Accordingly, we suggest that inter-unit measurement variability be investigated in future studies [9,24]. Finally, we acknowledge that the current findings are related to the specific GPS devices used in the present study. Accordingly, caution should be taken when applying the present findings when using alternate software or GPS hardware not examined in this investigation.

In conclusion, GPS accuracy increased with a higher sampling rate, but decreased with increasing speed. Collectively, the present results showed that GPS $-10 \mathrm{~Hz}$ is more accurate than GPS $-5 \mathrm{~Hz}$ for measuring distance covered during high-speed phases of intermittent running, while there are several concerns related to the use of both GPS devices to measure very highspeed running distances. The present study also showed that both $5 \mathrm{~Hz}$ and $10 \mathrm{~Hz}$ GPS could be used for calculating the mean metabolic power during high-intensity activities, whereas only the $10 \mathrm{~Hz}$ GPS demonstrated a sufficient level of accuracy for quantifying the time spent at HSR or at very high-power output. This is particularly relevant for accurately monitoring the true demands of intermittent exercise in order to develop sport-specific training programs aimed at improving physical performance and reducing injuries [10]. However, caution should be taken in the interpretation of the confidence intervals calculated in the present study. Indeed, the nature of the sample data used may have affected the variability of the data (possible effect of pseudoreplication), resulting in smaller confidence intervals. Furthermore, another limitation of the present project is that the exercise protocols used only change of speed and included only $180^{\circ}$ changes of direction. For these reasons it is difficult to directly generalize the present findings to team sport match play. Further studies with non-linear movements, larger sample sizes and using more specific team sport match simulations are necessary to fully validate GPS tracking systems.

\section{Acknowledgements}

$\nabla$

The authors would like to thank Andrea Bosio, Michele Tornaghi and Domenico Carlomagno for the support in the data collection and to Laura Garvican for her English revision. The authors would like also to thank all the athletes involved in the study.

Conflict of interest: The authors have no conflict of interest to declare. 
Affiliations

1 S. S. MAPEI srl, Human Performance Laboratory, Olgiate Olona, Italy ${ }^{2}$ Department of Biomedical Sciences for Health, Università degli Studi di Milano, Milano, Italy

${ }^{3}$ Faculty of Exercise Sciences, Department of Sport, Università degli Studi di Milano, Nutrition and Health Sciences, Milan, Italy

${ }^{4}$ S. S. MAPEI srl, Human Performance Laboratory, Castellanza, Italy

${ }^{5}$ Training Check Juventus, Juventus Football Club, Turin, Italy

${ }^{6}$ School of Leisure, Sport \& Tourism, University of Technology, Sydney,

Australia

School of Leisure, Sport \& Tourism, University of Technology, Sydney,

Lindfield, Australia

\section{References}

1 Aughey RJ. Applications of GPS technologies to field sports. Int J Sports Physiol Perform 2011; 6: 295-310

2 Bradley PS, Sheldon W, Wooster B, Olsen P, Boanas P, Krustrup P. High-intensity running in English FA Premier League soccer matches. J Sports Sci 2009; 27: 159-168

3 Castellano J, Casamichana D, Calleja-Gonzalez J, San Roman J, Ostojic SM. Reliability and accuracy of $10 \mathrm{~Hz}$ GPS devices for short-distance exercise. J Sports Sci Med 2011; 10: 233-234

4 Chelly SM, Denis $C$. Leg power and hopping stiffness: relationship with sprint running performance. Med Sci Sports 2001; 33: 326-333

5 Chelly SM, Hermassi S, Shephard RJ. Relationships between power and strength of the upper and lower limb muscles and throwing velocity in male handball players. J Strength Cond Res 2010; 24: 1480-1487

6 Cooper HJ, Hedges LV. The Handbook of research synthesis. New York: Russell Sage Foundation, 1994

7 Coutts AJ, Duffield R. Validity and reliability of GPS devices for measuring movement demands of team sports. J Sci Med Sport 2010; 13: 133-135

8 di Prampero PE, Fusi S, Sepulcri L, Morin JB, Belli A, Antonutto G. Sprint running: a new energetic approach. J Exp Biol 2005; 208: 2809-2816

9 Duffield R, Reid M, Baker J, Spratford W. Accuracy and reliability of GPS devices for measurement of movement patterns in confined spaces for court-based sports. J Sci Med Sport 2010; 13: 523-525

10 Gaudino P, Iaia FM, Alberti G, Strudwick AJ, Atkinson G, Gregson W. Monitoring training in elite soccer players: systematic bias between running speed and metabolic power data. Int J Sports Med 2013; 34: 963-968

11 Gray AJ, Jenkins $D$, Andrews $M H$, Taafe $D R$, Glover $M L$. Validity and reliability of GPS for measuring distance travelled in field-based team sports. J Sports Sci 2010; 28: 1319-1325
12 Harley JA, Lovell RJ, Barnes CA, Portas MD, Weston M. The interchangeability of global positioning system and semiautomated video-based performance data during elite soccer match play. J Strength Cond Res 2011; 25: 2334-2336

13 Harris DJ, Atkinson G. Ethical standards in sports and exercise research: 2014 update. Int J Sports Med 2013; 34: 1025-1028

14 Hopkins WG. Measures of reliability in sports medicine and science. Sports Med 2000; 30: 1-15

15 Hopkins WG. A scale of magnitudes for effect statistics Accessed 2002

16 Jennings D, Cormack S, Coutts AJ, Boyd L, Aurghey RJ. The validity and reliability of GPS units for measuring distance in team sport specific running patterns. Int J Sports Physiol Perform 2010; 5: 328-341

17 Little T, Williams A. Specificity of acceleration, maximum speed, and agility in professional soccer players. J Strength Cond Res 2005; 19 : 76-79

18 Mohr M, Krustrup P, Bangsbo J. Fatigue in soccer: a brief review. J Sports Sci 2005; 23: 593-599

19 Mohr M, Krustrup P, Bangsbo J. Match performance of high-standard soccer players with special reference to development of fatigue. J Sports Sci 2003; 21: 519-528

20 Osgnach C, Poser S, Bernardini R, Rinaldo R, di Prampero PE. Energy cost and metabolic power in elite soccer: a new match analysis approach. Med Sci Sports Exerc 2010; 42: 170-178

21 Petersen C, Pyne D, Portus M, Dawson B. Validity and reliability of GPS units to monitor cricket-specific movement patterns. Int J Sports Physiol Perform 2009; 4: 381-393

22 Rampinini E, Bishop D, Marcora SM, Ferrari Bravo D, Sassi $R$, Impellizzeri $F M$. Validity of simple field tests as indicators of match-related physical performance in top-level professional soccer players. Int J Sports Med 2007; 28: 228-235

23 Randers MB, Mujika I, Hewitt A, Santisteban J, Bischoff R, Solano $R$, Zubilaga A, Peltola E, Krustrup P, Mohr M. Application of four different football match analysis systems: a comparative study. J Sports Sci 2010; 28: 171-182

24 Varley MC, Fairweather IH, Aughey RJ. Validity and reliability of GPS for measuring instantaneous velocity during acceleration, deceleration, and constant motion. J Sports Sci 2012; 30: 121-127

25 Varley MC, FI H, Aughey RJ. Validity and reliability of GPS for measuring instantaneous velocity during acceleration, deceleration, and constant motion. J Sports Sci 2012; 30: 121-127 